\title{
Non-relativistic hollow electron beam formation for mm-wave BWO
}

\author{
V.L. Bratman ${ }^{1,2}$, A.E. Fedotov ${ }^{1}$, P.B. Makhalov ${ }^{1,3}$, and V.N. Manuilov ${ }^{1,4}$ \\ ${ }^{1}$ Institute of Applied Physics of the RAS, Nizhny Novgorod, Russia, fedotov@appl.sci-nnov.ru \\ ${ }^{2}$ Ariel University, Ariel, Israel \\ ${ }^{3}$ KTH University, Stockholm, Sweden \\ ${ }^{4}$ Nizhny Novgorod State University, Nizhny Novgorod, Russia
}

Slow-wave devices such as traveling-wave tubes (TWTs), backward-wave oscillators (BWOs), and extended-interaction klystrons (EIKs) are widely used as medium-power sources of short-millimeter and subterahertz waves. One of the difficulties that limits the $\mathrm{CW}$ or average power of slow-wave devices at short waves is partial interception of the electron beams by fragile slowwave structures (SWS) causing dangerous heating of the latter. In order to increase the power, the use of spatially distributed electron beams, mostly sheet beams, is widely studied last few years $[1,2]$. Another feasible and maybe somewhat simpler configuration is hollow rectilinear thin-wall electron beams interacting with the slow waves of azimuthally-symmetric operating waveguides [3, 4] similar to that of relativistic high-current microwave oscillators. Like in the case of sheet beams, the use of hollow electron beams permits a significant decrease in current density and heating of the microwave structure. To study this option, a W-band BWO with operating voltage of 30-32 kV has been designed in the Institute of Applied Physics. Oscillator operates at the lower axi-symmetric $\mathrm{TM}_{0,1}$ mode of microwave structure with a rectangular corrugation with following parameters: the period is $0.8 \mathrm{~mm}$, the inner diameter is $1.8 \mathrm{~mm}$ and the outer radius is $2.71 \mathrm{~mm}$. Simulations based on the PIC-code predicts sub-kW level of output power for W-band BWOoscillator with 1 A current.

The formation of a hollow thin-wall electron beam is a critical point for the further experimental realization of the slow-wave devices discussed above. Since the beam particles travel very close to the corrugated surface, strict requirements on transverse pulsations of electrons should be imposed. Namely, for guiding the beam at a distance of $0.1 \mathrm{~mm}$ from the corrugation, parasitic transverse velocities should not exceed $0.05 \mathrm{c}$. To drive the W-band BWO, the $30 \mathrm{kV} / 1$ A hollow electron beam with an outer diameter of 1.6 was designed. The beam is formed in Pierce-like electron gun with thermionic cathode and moderate emission density of $2 \mathrm{~A} / \mathrm{cm}^{2}$. To increase the current density, a 100-fold compression in an increasing magnetic field is used which leads to a tenfold increase in parasitic transverse velocities. The magnetic field is produced by main solenoid and cathode coil with reverse polarity. In the interaction region, the beam is guided in the magnetic field of $0.7 \mathrm{~T}$.

Numerical simulations of the gun were carried out using 2-D code EPOS [5] and 3-D PIC-code CST Studio. In order to diminish the beam edge pulsations, the cathode geometry was optimized to provide very accurate beam formation, see Fig. 1. Simulations predict the beam wall thickness of $0.1 \mathrm{~mm}$ in the interaction region and small radii of cyclotron rotations of particles (Fig. 2). The particles at the outer edge of hollow beam have smaller cyclotron radii than at the inner edge, which is favorable for the beam guiding. According to these results, electrons can be guided at the distance of $0.08 \mathrm{~mm}$ from SWS wall. In simulations, parasitic cyclotron rotation mainly occurs due to the initial electron transverse velocities arising from the thermal electron motion and emitter surface roughness.

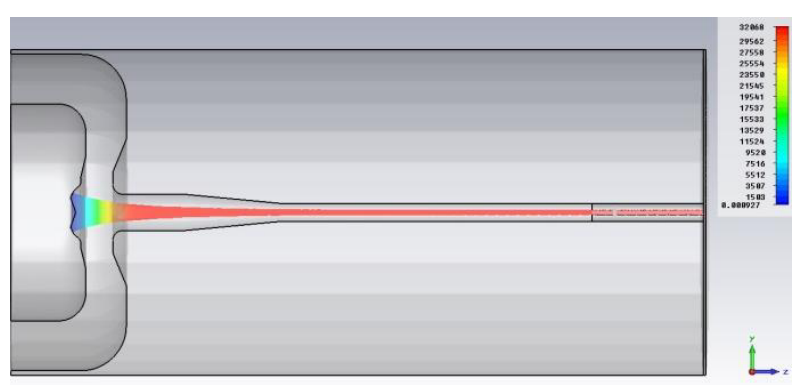

Fig. 1. Electron gun geometry and particles trajectories simulated by CST Studio code

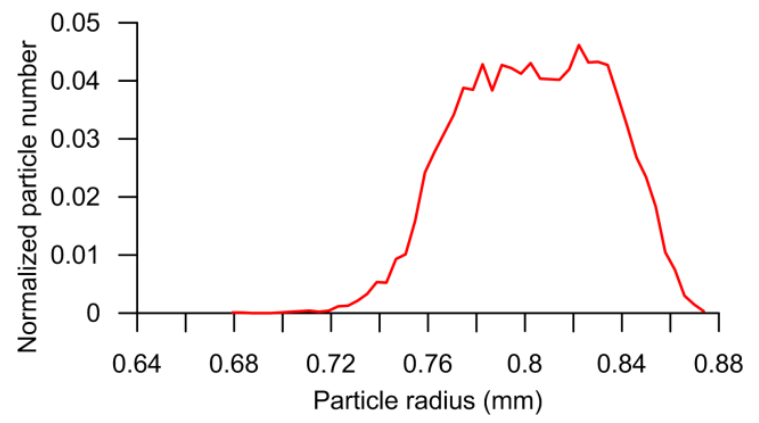

Fig. 2. Simulated distribution of electrons over radius in slow-wave structure

In the preliminary electron-optical experiment, 1-A hollow beam was formed. The outer beam radius could be varied by changing the magnetic compression ratio. For the designed parameters, $0.55 \mathrm{~A}$ current was guided through the slow-wave structure with inner radius of $0.9 \mathrm{~mm}$ while $0.45 \mathrm{~A}$ were intercepted by cutoff narrowing at the cathode side of the structure, see Fig. 3.

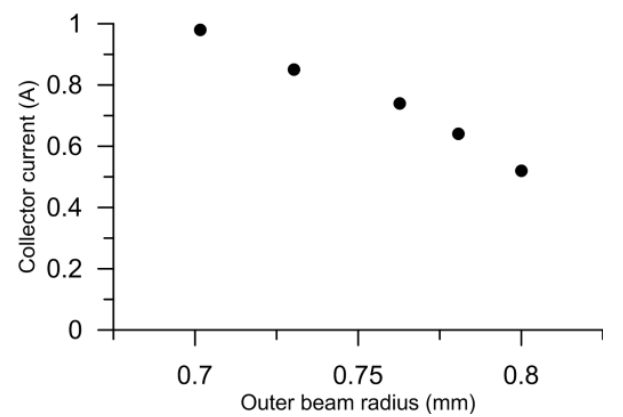

Fig. 3. Measured collector current vs. outer beam radius 
Thus, in the experiment, the beam wall thickness of about $0.3 \mathrm{~mm}$ was measured while simulations predict beam wall thickness of $0.15 \mathrm{~mm}$. The difference between simulations and experiment can be caused, in principle, by a number of reasons. One of the reasons is the possible misalignment of components of tube and magnetic system. Another probable reason is a cathode surface roughness which could result in an increased velocity spread of electrons. To study this effect, we have analyzed the cathode under microscope, see Fig. 4. The shape of the emitter surface was found numerically using a series of microscope photos made with different distance between the objective and the cathode. Then, this obtained shape was used in direct numerical simulations of the electron motion near the emitter surface made using 3-D PICcode. Results of the simulations (Fig. 5) predict that emitter surface roughness could not be the main reason for the beam interception. These results are in good agreement with theoretical estimations based on known formulas. Therefore, we conclude that the misalignment of the solenoid and the cathode coil, on the one side, and the anode, cathode, and slow-wave structure, on the other side, is the most probable reason for the beam interception, so efforts will be made to minimize these effects in the following experiments.
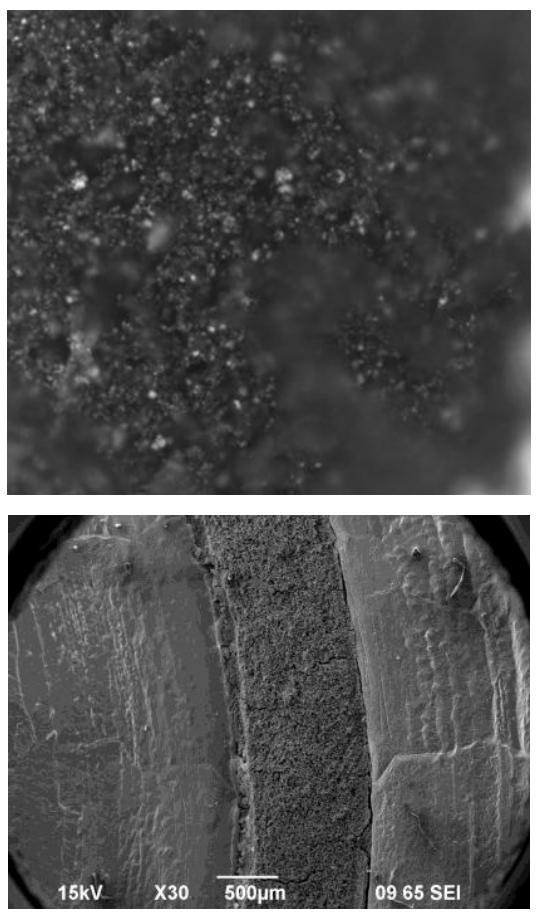

Fig. 4. Microphotographs of emitter surface using light and electronic microscopes

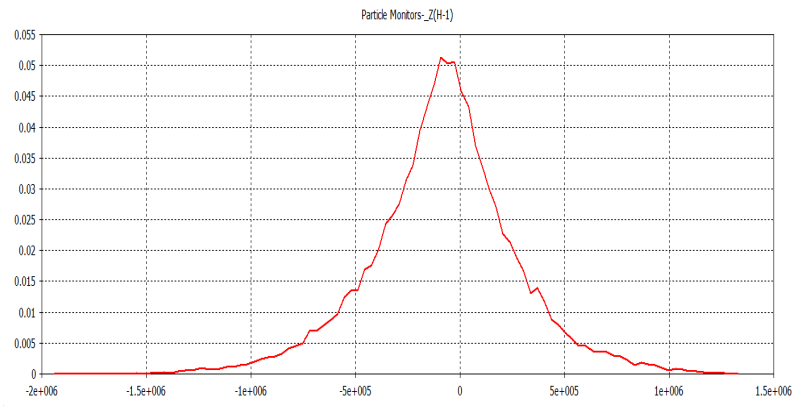

Fig. 5. Simulated distribution of parasitic electron transverse velocities caused by emitter surface roughness

Simulations of electron-wave interaction based on the averaged equations and 3D PIC-code CST predict that BWO with the existing hollow beam can provide output power up to $300 \mathrm{~W}$ (Fig. 6) and wideband frequency tuning.

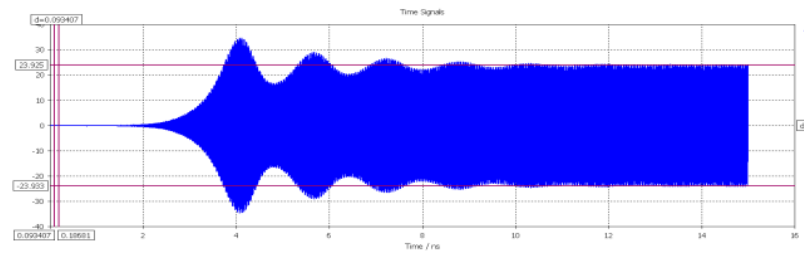

Fig. 6. Simulated output signal for BWO with 0.7 A hollow electron beam with wall thickness of $0.3 \mathrm{~mm}$ 01174.

The work is supported by RFBR, project No. 16-08-

\section{References}

1. Y.-M. Shin, J.-X. Wang, L.R. Barnett, and N.C. Luhmann, $J r$. Particle-In-Cell simulation analysis of a multicavity W-band sheet beam klystron. // IEEE Trans. Electron Dev. 2011. V. 58, No. 1. P. 251-258.

2. J. Lai, Y. Gong, X. Xu, Y. Wei, Z. Duan, W. Wang, and J. Feng. W-band 1-kW staggered double-vane traveling-wave tube. IEEE Trans. Electron Dev. 2012. V. 59, No. 2. P. 496-503.

3. V.L. Bratman, A.E. Fedotov, and P.B. Makhalov. Highfrequency devices with weakly-relativistic hollow thin-wall electron beams. // Physics of Plasmas. 2012. V. 19, No. 2. Art. 020704.

4. V.L. Bratman, A.E. Fedotov, P.B. Makhalov, and V.N. Manuilov. Design and numerical analysis of W-band oscillators with hollow electron beam. // IEEE Transactions on Electron Devices. 2014. Vol. 61, No. 6. P. 1795-1799.

5. P.V. Krivosheev, V.K. Lygin, V.N. Manuilov, and Sh.E. Tsimring. Numerical simulation models of forming systems of intense gyrotron helical electron beams. // Int. J. Infrared and Millimeter Waves. 2001. V. 22, N. 8. P. 1119-1145. 